\title{
Editorial
}

\section{Modern Phased Arrays and Their Hybrid Intelligent Processing}

\author{
Chung-Liang Chang, ${ }^{1}$ Juin J. Liou, ${ }^{2}$ Hon Tat Hui, ${ }^{3}$ and Chiao-En Chen ${ }^{4}$ \\ ${ }^{1}$ Department of Biomechatronics Engineering, National Pingtung University of Science and Technology, Pingtung County 91201, Taiwan \\ ${ }^{2}$ Department of Electrical Engineering and Computer Science, University of Central Florida, Orlando, FL 32816, USA \\ ${ }^{3}$ Department of Electrical and Computer Engineering, National University of Singapore, Singapore 117576 \\ ${ }^{4}$ Department of Electrical Engineering/Communication Engineering, National Chung Cheng University, Chiayi County 621, Taiwan
}

Correspondence should be addressed to Chung-Liang Chang; chungliang@mail.npust.edu.tw

Received 4 May 2014; Accepted 4 May 2014; Published 25 June 2014

Copyright (C) 2014 Chung-Liang Chang et al. This is an open access article distributed under the Creative Commons Attribution License, which permits unrestricted use, distribution, and reproduction in any medium, provided the original work is properly cited.

Recently, intelligent methods, including fuzzy inference, robust control, evolutionary computation, swarm intelligence, and ant colony, play an important role in the development of smart sensor array technology, which brings together additional interdisciplinary research skills in the development of innovative antijam devices, microelectromechanical systems, aerospace, laser, optics, wireless communications, and multiple signal reception processor for modern phased array applications. One of the main advantages of using phased array processing along with an array of sensors is a smaller footprint. The problems associated with array processing include the number of sources used, their direction of arrivals, sensor placement, sensor structure, and their signal waveforms. Generally, satisfactory results can be obtained by employing conventional intelligent algorithms on smart sensor array applications.

However, smart antenna array technologies demand to be more robust, have more precision, be costly, and be more efficient computation complexity; therefore, it is worthy of our effort to develop hybrid intelligent algorithms and mechanical solutions capable of improving the performance of existing sensor array technology. The current research orients towards low-cost and simple methods to overcome the effect of uncertainties on the whole array system. In particular, after the future array system is miniaturization, the design method of circuit protection within array system is inevitably a crucial issue. Recent research illustrates that hybrid intelligent methods which are integrated with some standard ones can help achieve a better performance for particular phased arrays applications. It was indicated that hybrid intelligent processing can generally bring a more convincing solution for a particular micro/nanoelectronics array application.

The goal of this special issue is to bring together researchers to highlight the innovative results or guidelines of future research directions in the development and implementation of hybrid intelligent processing concerning modern phased array. Application articles listed in this special issue are innovative and thought provoking. Numerical simulations and experimental validation have been implemented. Several sensor array devices have already been developed and performance has been recorded. In the following, we present an overview of the articles of the special issue.

In the field of antenna array system design, three articles have been published; S. Nako et al. propose a wireless power transfer system adopting a microwave active phased array. The results demonstrate that the axial ration of $0.44 \mathrm{~dB}$ is achieved with power fluctuation being kept below $1 \%$, while K. R. Subhashini and A. T. P. Kumar employ adaptive based differential evolution (ADE) and fire fly (AFA) algorithm to determine a set of parameters of antenna elements that provide the desired radiation pattern. The results indicate nonlinear synthesis, which offers tremendous improvements in contrast to linear synthesis using the techniques mentioned in their paper; C.-L. Chang et al. present a compressive sensing based array processing method to reduce the complexity and the computation load of array system and to maintain the robust antijam performance in global navigation satellite system (GNSS) receiver. Their theoretical analysis result using the proposed algorithm is verified based on simulation results. 
In addition, there are two articles about the discussion of stability of nonlinear system in the presence of uncertainty; H.-N. Shou's paper will be the first investigation to UKF microsatellites in LEO orbit in real time, which attempts to discover the real-time precision orbit determination techniques. It is presented that, based on orbit mission requirements and conditions using UKF, they can satisfy the positioning accuracy and compute two indicators; Y.-Y. Hou and Z.-L. Wan investigate into the problem of the robust stability for the nonlinear system with time-varying delay and parameters uncertainties. The numerical simulations demonstrate the performance and feasibility of proposed methods.

Regarding phased array application and system implementation, N. Mishra et al. propose a novel knowledge discovery strategy and an exploration algorithm for indoor safety-critical sensor applications. Their proposed strategy and algorithm can be implemented for knowledge discovery within the operational framework; J.-S. Sheu et al. develop a method using a three-axis accelerometer and a singlechip microcontrol unit to conduct a three-dimensional (3D) pedometer capable of automatically identifying walking and running motions. The proposed technique can remedy drawbacks of generic mechanical and 3D pedometers; the development of wireless communication platform field is explored by C.-S. Chen et al. This article researches the function and algorithm of the high definition radio frequency modulation digital radio broadcasting system. With the implementation of this system on the field programmable gate array (FPGA), based on hardware platform, theoretical and practical treatments are taken into account to accommodate the available hardware resources.

Finally, a sensing method asymmetrical fiber-optic MachZehnder interferometer (FO-MZI) is developed by C.-H. Chen et al. This paper is innovative in its adoption of a production process and cost-efficient method to develop an asymmetrical fiber-optic (FO) Mach-Zehnder interferometer (MZI) with sensing abilities. The MZI is combined with a small fiber-optic waveguide component to form an interferometer with fiber-optic characteristics. This results in a FOMZI.

\section{Acknowledgments}

Thanks are due to the contribution of articles by profound experts in the field of modern sensor array processing; we hope that those papers serve as a useful medium for future research projects and innovative incentive for future exploration. We want to express our greatest gratitude to each author for contributing such an inspiring research work to this special issue and also to all reviewers for their concentrated effort to enhance the quality of this special issue. It is such an honor and a pleasant experience for us to work on this special issue.

Chung-Liang Chang

Juin J. Liou

Hon Tat Hui

Chiao-En Chen 

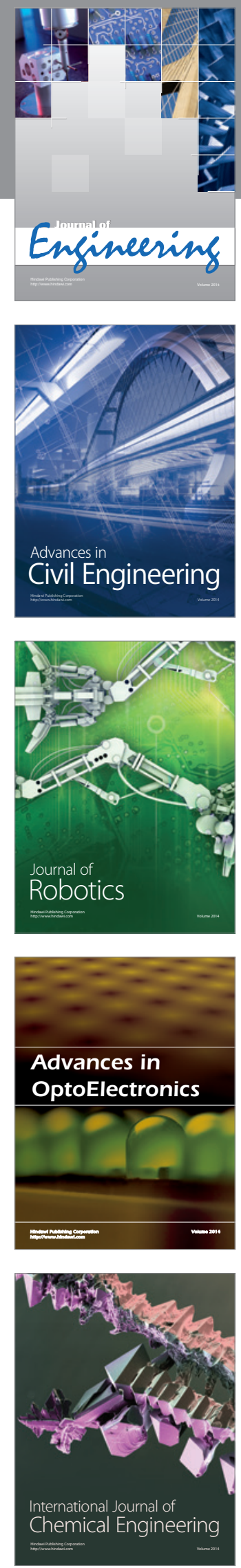

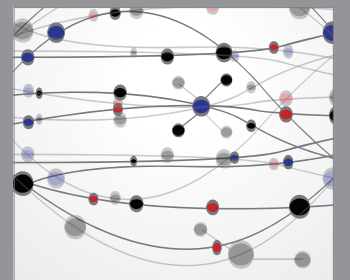

The Scientific World Journal
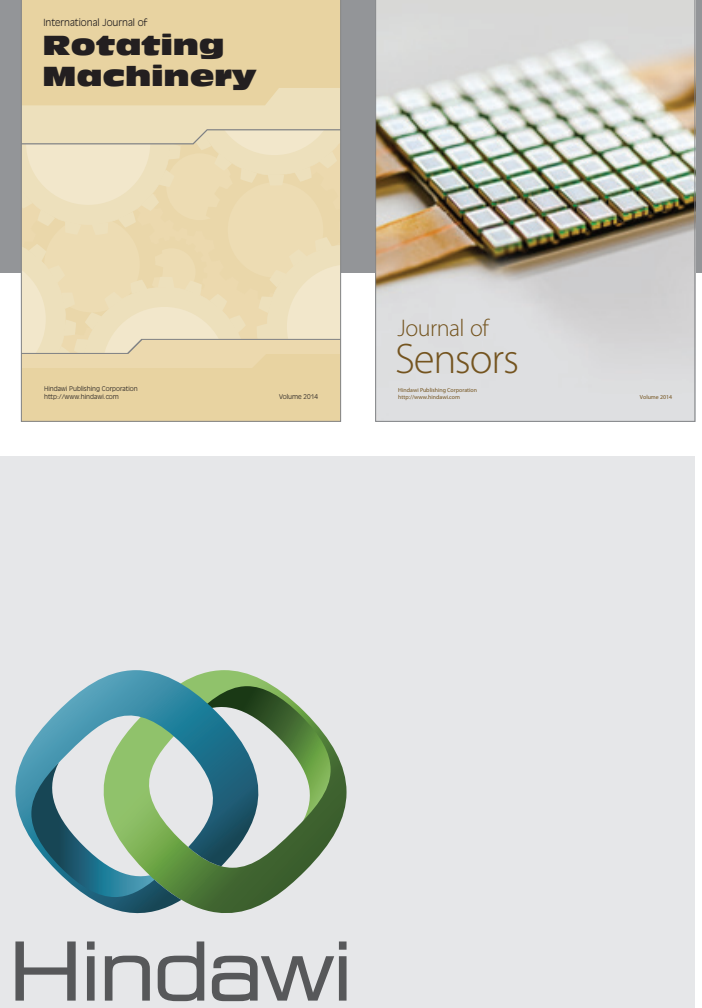

Submit your manuscripts at http://www.hindawi.com
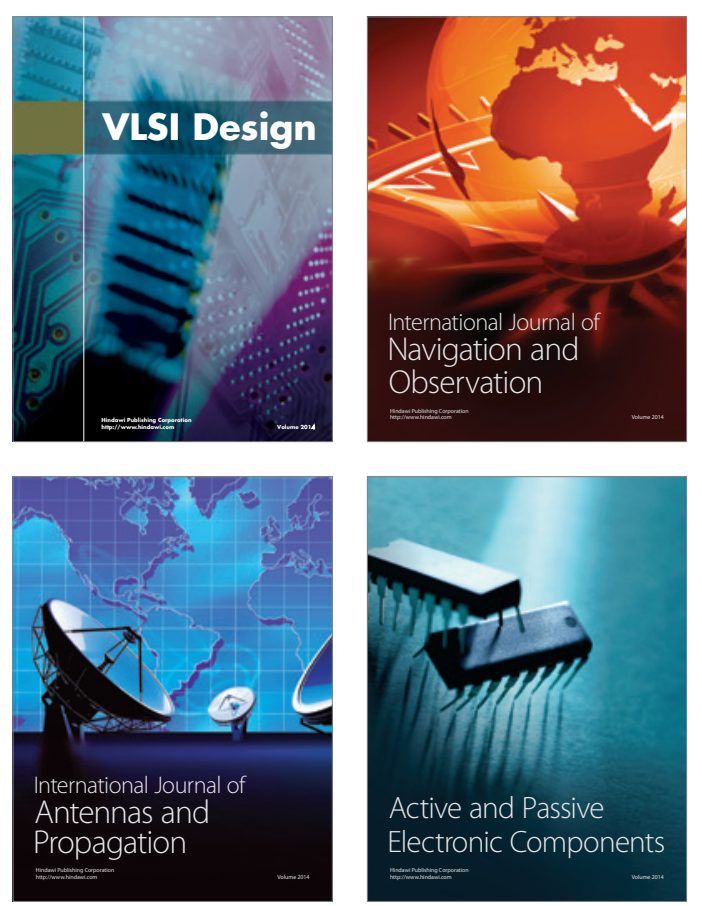
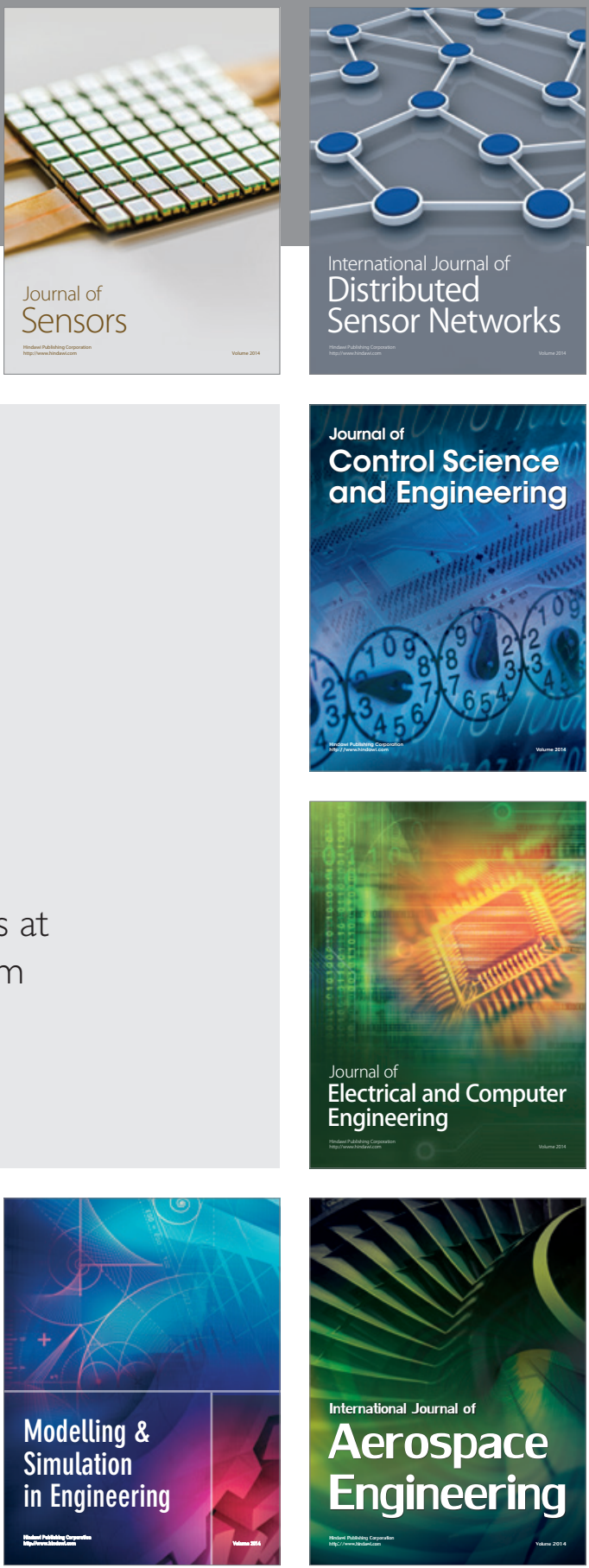

Journal of

Control Science

and Engineering
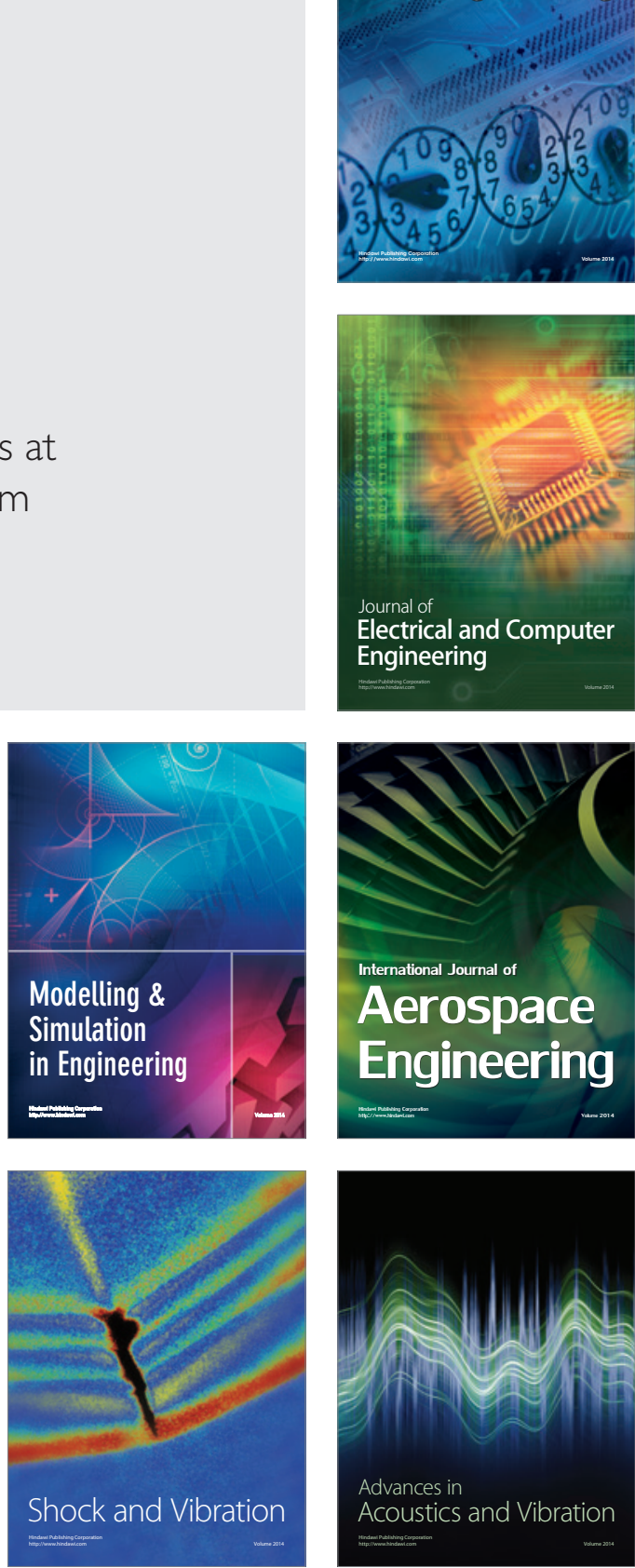